\title{
Is obesity all about excess food and sedentary lifestyle?
}

\begin{abstract}
Obesity has been on the rise over the years leading to an global epidemic. Policies and preventive strategies have been developed and disseminated focusing on the declared main contributors of obesity; overconsumption of convenient food and lack of physical activity. However it is easy to overlook the fact that obesity is a complex disease and one needs to understand the foundations causing this condition. Underlying cofounding factors need to be uncovered and understood before obesity management is initiated.
\end{abstract}

Keywords: obesity, epidemics, policy, life style
Volume 6 Issue 6 - 2017

Sarah Cuschieri
Department of Anatomy, University of Malta, Malta

Correspondence: Sarah Cuschieri, Department of Anatomy, University of Malta, Malta, Tel +356 794I5298,

Email sarah.cuschieri@um.edu.mt

Received: May 04, 2017 | Published: June 05, 2017

\section{Introduction}

Obesity is a well-established global epidemic. Recently the World Obesity Federation declared obesity as being a chronic relapsing disease. ${ }^{1}$ The commonest causes for the development of obesity have been attributed to overconsumption of convenient foods as well as the decline in physical activity. ${ }^{2,3}$ It is common practice among people and professionals alike that all obese individuals are stigmatized as being lazy and overeats. Conversely a large proportion of obese individuals declare that they try to be compliant with the obesity management and advise provided. This is easily dismissed and disregarded by professionals. However obesity is more than meets the eye. One needs to be open minded and understands the reason as to "Why" the individual is obese. ${ }^{4}$ How can we judge and criticize before we understand?

In some cultures obesity is the norm and their surrounding environment may not provide the right psychological and biological bases for the maintenance of obesity management. The mental state needs to be evaluated prior to initiating obesity management, since mental health acts as both a comorbidity and as a barrier. Without psychological aid these obese individuals will never reach their management potential.

Another issue to be considered in that most obese individuals have underlying comorbidities, which act as a situation of "which came first, the chicken or the egg"?. Let's take osteoarthritis as an example of a common disease among individuals. The presence of knee or hip arthritis will limit the individual from easy mobility and as a consequence reduce the amount of physical activity that could be tolerated, trigging obesity. The situation may be the other way round; the obese individual develops knee arthritis over time due to the increased weight on the weight bearing joints, causing distress and limitation of movement. This will impact on the management of obesity especially performing physical activity. There are other mechanical disorders that also pose barriers to the required increase in physical activity and maintaining obesity management such as urinary incontinence or obstructive sleep apnea.

There are metabolic disorders that inevitably lead to an increase in weight and consequently development of obesity such as in polycystic ovarian syndrome (PCOS) and Cushing's syndrome. In which cases, it is important for the caring physician to pick up the other corresponding signs and symptoms and diagnose such conditions. Reduction in body weight will improve these conditions. Also maintaining the correct management plan for these conditions will lead to weight loss.

Leading a healthy lifestyle is not a walk in the park. Healthy food including fruit and vegetables are relatively more expensive when compared to convenient unhealthy food. Acquiring a life or medical insurance is also another financial burden. Although not every country enjoys free health service, those that do are still faced with long waiting lists and appointments to be seen by a health professional, be it a specialist doctor or a nutritionist. Getting a quicker service means the individual needs to seek private services. Hence, one needs to keep in mind that some obese individuals may be empowered to lead a healthy lifestyle but are not financially capable to do so, since maintain a healthy lifestyle is expensive.

\section{Conclusion}

Everyone agrees that obesity is a huge burden and action needs to be taken but there are many cofounding factors that need to be considered before anything can be done. Understanding the individual holistically is the key and not just perceiving the individual as a ticking bomb waiting to explode with complications and pertaining an economical burden. It is useless to set up strategies and policies on paper without grasping the underlying issues since we will hit a standstill, claiming that obesity is an epidemic without taking appropriate action!

\section{Acknowledgements}

None.

\section{Conflict of interest}

The author declares no conflict of interest.

\section{References}

1. Bray GA, Kim KK, Wilding JPH. Obesity: a chronic relapsing progressive disease process. A position statement of the World Obesity Federation. Obesity reviews. 2017.

2. Scully T. New insights for obesity biology. Nature. 2015;518:197-206.

3. Bray GA, Wadden TA. Improving long-term weight loss maintenance: can we do it? Obesity. 2015;23:2-3.

4. Sharma AM. M,M,M \&M: a mnemonic for assessing obesity. Obesity reviews. 2010;11(11):809-809. 